\title{
Bounds on a Distribution Function That Are Functions of Moments to Order Four ${ }^{1}$
}

\author{
Marvin Zelen
}

Explicit expressions are presented for bounds on a distribution function when moments to order four are known. These inequalities are given in a form suitable for applications.

\section{Introduction and Statement of Problem}

Tchebycheff [7] ${ }^{2}$ in 1874 proposed a problem that can be stated as follows: Let $F(y)$ be an unknown distribution function over the closed interval ${ }^{3}[a, b]$, and satisfying the conditions

$$
\begin{gathered}
F(a-0)=0 \\
m_{j}=\int_{a}^{b} y^{j} d F^{\prime}(y) \quad(j=0,1, \ldots, k) .
\end{gathered}
$$

If the moments $m_{j}$ for $j=0,1, \ldots, k$ are known, then for a given value of $x,(a<x<b)$, what are the (sharp) upper and lower bounds on $F(x)$ ?

Tchebycheff presented without proof a solution to the above problem, which is sometimes called the reduced-moment problem. Proofs were later given by Markoff [1], Possé [2], and Stieltjes [5,6]. The book by Shohat and Tamarkin [4] gives an account of some of the modernday treatments of the subject.

This paper presents the explicit expressions for solutions of the moment problem (often referred to as the Tchebycheff-Markoff inequalities) for the cases $k=2,3,4$. Inequalities that are functions of moments to order two were given by Tchebycheff [7] for distributions over the interval $[0, b]$. Inequalities that are functions of moments to order three were given by Possé [2]. Possé also solved the case of four moments for distributions over the interval $[a, \infty)$.

\section{Explicit Expressions for Bounds}

This section presents without proof the explicit expressions for bounds on a distribution function. Proofs may be found in [9]. These are derived as special cases of the TchebycheffMarkoff inequalities.

In all that follows it will be assumed that

$$
m_{0}=1, \quad m_{1}=0, \quad m_{2}=1 .
$$

This will result in no loss of generality, as any distribution function can be made to conform to these conditions by the use of a linear transformation. The assumption (1) implies that $a, b$ satisfy the inequalities

$$
\left.\begin{array}{l}
a<0<b \\
1+a b \leq 0 .
\end{array}\right\}
$$

This follows from the necessary conditions for the solution of the moment problem (cf. Shohat and Tamarkin [4]).

\footnotetext{
1 A condensation of certain results obtained by the author in a thesis submitted to the University of North Carolina in June 1951 in partial fulfillment of the requirements for the Master of Arts degree.

2 Figures in brackets indicate the literature references at the end of this paper.

3 Throughout this paper it will be understood that a distribution function over the interval $[a, b]$ is one where the range of the random variable is $[a, b]$, and the end points will belong to that interval unless the end points are $-\infty$ or $+\infty$.
} 


\subsection{Bounds for Two Moments}

Let $F(y)$ be a distribution function on $[a, b]$ with known moments

$$
m_{0}=1, \quad m_{1}=0, \quad m_{2}=1
$$

then for a given $x,(a<x<b)$,

$$
\begin{array}{ccrl}
0 & \leq F(x) \leq \frac{1}{1+x^{2}} & & \text { if } a<x \leq-\frac{1}{b} \\
\frac{1+b x}{(a-b)(a-x)} & \leq F(x) \leq 1-\frac{1+a x}{(b-a)(b-x)} & & \text { if }-\frac{1}{b} \leq x \leq-\frac{1}{a} \\
\frac{x^{2}}{1+x^{2}} & \leq F(x) \leq 1 & & \text { if }-\frac{1}{a} \leq x<b .
\end{array}
$$

For any distribution defined over $(-\infty, \infty)$ inequalities (3) and (5) hold for $x<0$ and $x>0$, respectively.

\subsection{Bounds for Three Moments}

Let $F(y)$ be a distribution function on $[a, b]$ with known moments

$$
m_{0}=1, \quad m_{1}=0, \quad m_{2}=1, \quad m_{3} .
$$

Let $g(x)=x^{2}-m_{3} x-1$, and define

$$
\begin{aligned}
z_{1} & =\frac{m_{3}-(a+x)}{1+a x} \\
z_{2} & =\frac{m_{3}-(b+x)}{1+b x} \\
w & =\frac{m_{3}-(a+b)}{1+a b} \\
A(\alpha, \beta, \gamma) & =\frac{1+\alpha \beta}{(\gamma-\alpha)(\gamma-\beta)},
\end{aligned}
$$

then for a given $x,(a<x<b)$

$$
\begin{aligned}
0 & \leq F(x) \leq A\left(b, z_{2}, x\right) & & \text { if } x<0, g(x) \geq 0 \\
A\left(x, z_{1}, a\right) & \leq F(x) \leq A\left(x, z_{1}, a\right)+A\left(a, z_{1}, x\right) & & \text { if } g(x) \leq 0, x \leq w \\
A\left(x, b, z_{2}\right) & \leq F(x) \leq A\left(x, b, z_{2}\right)+A\left(b, z_{2}, x\right) & & \text { if } g(x) \leq 0, x \geq w \\
1-A\left(a, z_{1}, x\right) & \leq F(x) \leq 1 & & \text { if } g(x) \geq 0, x>0 .
\end{aligned}
$$

Inequalities (7) and (9) hold for any distribution $F(y)$ on $[a, \infty)$. Inequalities (6) and (8) hold for any distribution $F(y)$ on $(-\infty, b]$. Note that none of the inequalities (6) to (9) holds for distributions over $(-\infty, \infty)$.

\subsection{Bounds for Four Moments}

Let $F(y)$ be a distribution function on $[a, b]$ with moments

$$
m_{0}=1, \quad m_{1}=0, \quad m_{2}=1, \quad m_{3}, \quad m_{4} .
$$

Let

$$
g(y, \gamma)=y^{2}+\left[\frac{\gamma-m_{3}+\gamma\left(\gamma m_{3}-m_{4}\right)}{1+\gamma\left(m_{3}-\gamma\right)}\right] y+\left[\frac{\gamma m_{3}-m_{4}+\left(m_{3}-\gamma\right)^{2}}{1+\gamma\left(m_{3}-\gamma\right)}\right]
$$


$U(y)=g(y, a), V(y)=g(y, b), Z(y)=g(y, x)$, and let $u_{1}<u_{2}, v_{1}<v_{2}, z_{1}<z_{2}$ be the distinct zeros of $U(y), V(y), Z(y)$, respectively, then $a<v_{1}<u_{1}<v_{2}<u_{2}<b$.

Define

$$
\begin{aligned}
z_{3} & =\frac{m_{3}(a+b+x)-m_{4}-a b-a x-b x}{a b x+a+b+x-m_{3}} \\
A & =\frac{m_{4}-m_{3}^{2}-1}{\left(1+x^{2}\right)\left(m_{4}-m_{3}^{2}-1\right)+\left(x^{2}-m_{3} x-1\right)^{2}} \\
B(\alpha, \beta, \gamma) & =\frac{m_{3}-\left(\alpha+\beta+z_{3}\right)-\alpha \beta z_{3}}{(\gamma-\alpha)(\gamma-\beta)\left(\gamma-z_{3}\right)},
\end{aligned}
$$

then for a given value of $x,(a<x<b)$,

$$
\begin{array}{ccrl}
0 & \leq F(x) \leq A & & \text { if } a<x \leq v_{1} . \\
B(b, x, a) & \leq F(x) \leq B(b, x, a)+B(a, b, x) & & \text { if } v_{1} \leq x \leq u_{1} \\
\frac{1+x z_{2}}{\left(z_{1}-x\right)\left(z_{1}-z_{2}\right)} & \leq F(x) \leq \frac{1+x z_{2}}{\left(z_{1}-x\right)\left(z_{1}-z_{2}\right)}+A & & \text { if } u_{1} \leq x \leq v_{2} \\
1-B(a, b, x)-B(a, x, b) & \leq F(x) \leq 1-B(a, x, b) & & \text { if } v_{2} \leq x \leq u_{2} \\
1-A & \leq F(x) \leq 1 & & \text { if } u_{2} \leq x<b .
\end{array}
$$

For any distribution defined over $(-\infty, \infty)$ inequalities (10), (12), and (14) hold, respectively, for $x<z_{1}, z_{1}<x<z_{2}, z_{2}<x$.

However, the ordering of $x$ in relation to $z_{1}, z_{2}$ is equivalent to the following. Let $g(x)=x^{2}-m_{3} x-1$, then

$$
\begin{array}{cl}
x>0, \quad g(x)>0 & \text { if, and only if, } z_{2}<x . \\
x<0, \quad g(x)>0 & \text { if, and only if, } z_{1}>x \\
g(x)<0 & \text { if, and only if, } z_{1}<x<z_{2} .
\end{array}
$$

Using (15) to (17), the applications of the Tchebycheff-Markoff inequalities for the case where $F(y)$ is defined over $(-\infty, \infty)$ are made particularly easy.

\section{Application of the Tchebycheff-Markoff Inequalities}

Let $F(y)$ be a distribution function whose first four moments coincide with those of the standard normal distribution, i. e., $m_{0}=1, m_{1}=0, m_{2}=1, m_{3}=0, m_{4}=3$. The TchebycheffMarkoff inequalities will be used to find bounds for $F(x)$ when $x=2,3$.

Bounds using two moments: Since $x>0$, inequality (5) is applicable, and we have

$$
\begin{aligned}
& .8000 \leq F(2) \leq 1 \\
& .9000 \leq F(3) \leq 1 .
\end{aligned}
$$

Bounds using four moments: Since $x>0, g(2)>0, g(3)>0$, inequality (14) is applicable. Substituting the appropriate values, we have

$$
1-\frac{2}{2\left(1+x^{2}\right)+\left(x^{2}-1\right)^{2}} \leq F(x) \leq 1 \text { for } x=2,3
$$

therefore,

$$
\begin{aligned}
& .8947 \leq F(2) \leq 1 \\
& .9777 \leq F(3) \leq 1 .
\end{aligned}
$$

Note that there are no inequalities applicable using only moments to order three. 


\section{Appendix}

Statements and proofs of the Tchebycheff-Markoff inequalities can be found in Shohat and Tamarkin [4], Uspensky [8], and Royden [3]. This section contains a statement of the Tchebycheff-Markoff inequalities as the above sources do not give the theorem in full generality, and it is not readily available in the literature.

Before stating the theorem it will be convenient to define the following: Let $T_{n}(y), U_{n}(y)$, $V_{n}(y), W_{n}(y)$ be polynomials of degree $n$ defined by

$$
\begin{aligned}
& \int_{a}^{b} T_{n}(y) \theta_{n-1}(y) d F(y)=0 \\
& \int_{a}^{b} U_{n}(y) \theta_{n-1}(y)(y-a) d F(y)=0 \\
& \int_{a}^{b} V_{n}(y) \theta_{n-1}(y)(b-y) d F(y)=0 \\
& \int_{a}^{b} W_{n}(y) \theta_{n-1}(y)(y-a)(b-y) d F(y)=0,
\end{aligned}
$$

where $\theta_{n-1}(y)$ is any polynomial of degree $\leq n-1$, and the coefficient for $y^{n}$ in $T_{n}(y), U_{n}(y)$ $V_{n}(y), W_{n}(y)$ is unity.

Tchebycheff-Markoff Inequalities: Let $F(y)$ be any distribution function on $[a, b]$ with moments $m_{0}, m_{1}, \ldots, m_{k}$

$$
m_{j}=\int_{a}^{b} y^{j} d F(y) \quad(j=0,1, \ldots, k),
$$

and let $x$ be a given number $(a<x<b)$, then

where

$$
\sum_{y_{i}<x} \frac{p\left(y_{i}\right)}{q^{\prime}\left(y_{i}\right)} \leq F(x) \leq \sum_{y_{i}<x} \frac{p\left(y_{i}\right)}{q^{\prime}\left(y_{i}\right)}+\frac{p(x)}{q^{\prime}(x)}
$$

$$
p(z)=\int_{a}^{b} \frac{q(z)-q(y)}{z-y} d F(y)
$$

and $y_{1}<y_{2}<\ldots<x<\ldots$ are the zeros of the polynomial $q(y)$ of degree $r$ defined by

$$
\begin{array}{ll}
q(y)=(y-x) w(y) & \text { if } k=2 n, U_{n}(x) V_{n}(x)>0, \\
q(y)=(y-b)(y-a)(y-x) w(y) & \text { if } k=2 n, U_{n}(x) V_{n}(x)<0, \\
q(y)=(y-a)(y-x) w(y) & \text { if } k=2 n-1, T_{n}(x) W_{n-1}(x)>0, \\
q(y)=(y-b)(y-x) w(y) & \text { if } k=2 n-1, T_{n}(x) W_{n-1}(x)<0,
\end{array}
$$

where $r=n+2$ for eq (23), $r=n+1$ for (22), (24), (25), and $w(y)$ is determined by

$$
\begin{array}{ll}
\int_{a}^{b} q(y) y^{i} d F(y)=0 & i=0,1, \ldots, n-1 \text { for }(22) \\
& i=0,1, \ldots, n-2 \text { for }(23),(24),(25) .
\end{array}
$$

Corollary: For eq (22) the inequalities hold for any distribution over $(-\infty, \infty)$ with moments $m_{0}, m_{1}, \ldots, m_{2 n}$. The inequalities for eq (24) hold for any distribution over $[a, \infty)$ with moments $m_{0}, m_{1}, \ldots, m_{2 n-1}$. The inequalities for eq (25) hold for any distribution over $(-\infty, b]$ with. moments $m_{0}, m_{1}, \ldots, m_{2 n-1}$. 
I wish to express my thanks to Professor Wassily Hoeffding, University of North Carolina, for his invaluable guidance during the preparation of this work.

\section{References}

[1] A. Markoff, Démonstration de certaines inégalitiés de M. Tchebycheff, Math. Ann. XXIV, 172-180 (1884).

[2] C. Possé, Sur quelques applications des fractions continues algebriques (Academie Imperiale des Sciences, St. Petersburg, 1886).

[3] H. L. Royden, Bounds on a distribution function when its first $n$ moments are given, Ann. Math. Stat. 24, $361-376(1953)$.

[4] J. A. Shohat and J. D. Tamarkin. The problems of moments (Am. Math. Soc. New York, 1943).

[5] T. J. Stieltjes, Sur l'évaluation approchée des intégrales, Compt. rend XCVII, 740-742, 798-799 (1883).

[6] T. J. Stieltjes, Recherches sur les fractions continues, Ann. faculté sciences Toulouse VIII, 1-22 (1894); IX, 45-47 (1895)

[7] P. Tchebycheff, Sur les valeurs limites des intégrales, J. de Math. [2] XIX, 157-160 (1874).

[8] J. V. Uspensky, Introduction to mathematical probability (McGraw-Hill Book Co., New York, N. Y., 1937).

[9] M. Zelen, Bounds on a distribution function which are functions of moments, unpublished masters thesis (University of North Carolina Library, 1951).

Washington, November 20, 1953. 